\title{
Commissioning of the ATLAS offline software with cosmic rays
}
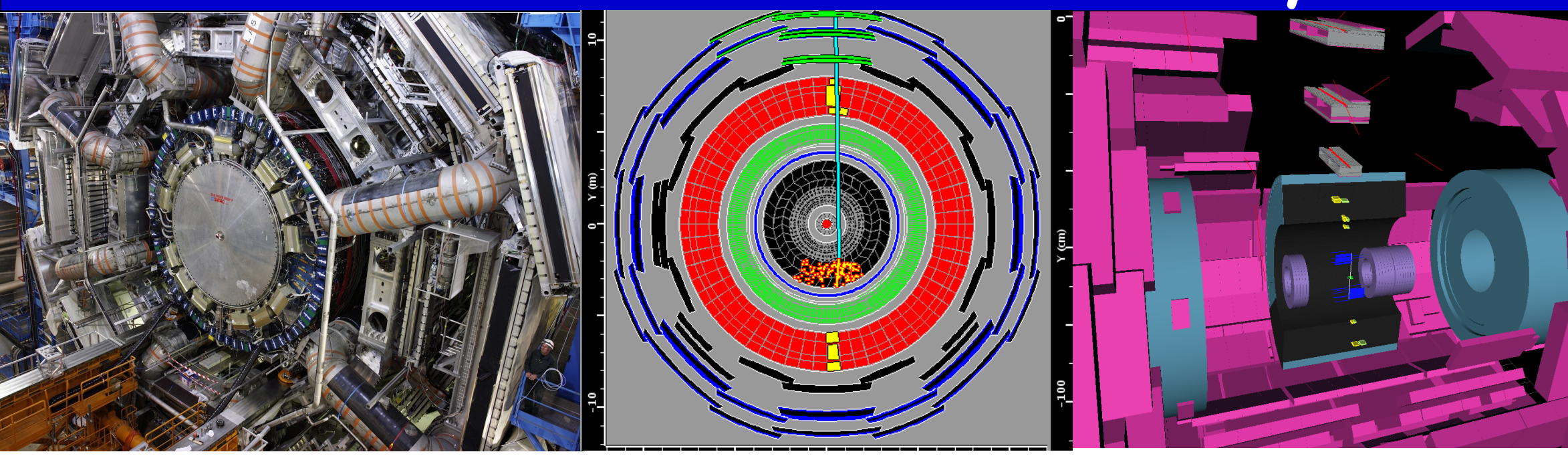

José Maneira (LIP-Lisboa) on behalf of the ATLAS offline commissioning group: IEEE/NSS - Honolulu - Oct. 30, 2007 
Introduction 


\section{Inner detector}

Transition Radiation

Tracker (TRT)

Straw tubes

$\sim 3.5^{\star} 10^{5}$ channels

Pixels

Si Pixel Detectors $\sim 0.8 * 10^{8}$ channels

- Related talks

- M. Keil, J. Weingarten (N26), S. Mima (N30), J.R. Schieck (N56)
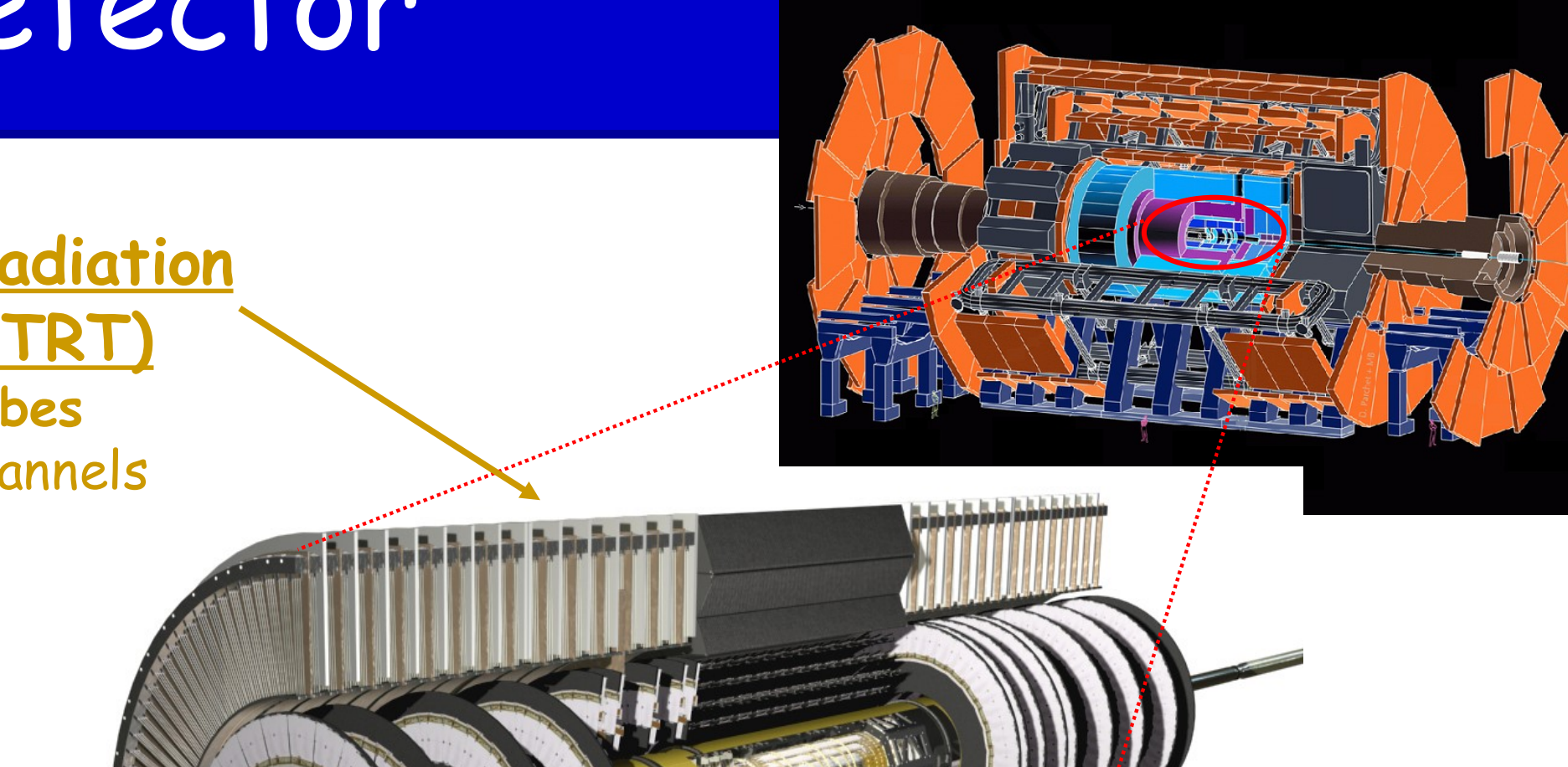

SemiConductor Tracker (SCT) Si Strip Detectors $\sim 6 * 10^{6}$ channels 


\section{Calorimeters}
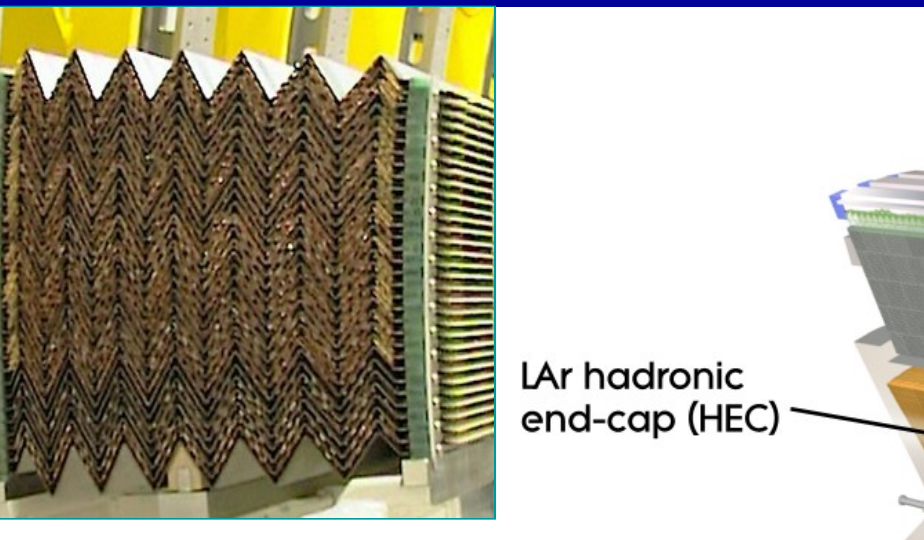

LAr electromagnetic end-cap (EMEC)

- Lead-Liquid argon calorimeters

- Accordion shape electrodes collect ionization charge

- Barrel in same cryostat as solenoid $\sim 2 \times 10^{5}$ channels

LAr electromagnetic barrel

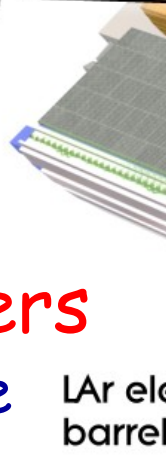

Related talk yesterday by D. Rousseau

- Hadronic Tile calorimeter

- Iron-scintillating tiles

- WLS fibres-PMTs readout

- 104 channels

\section{Scintillator}




\section{Muon spectrometer}

Trigger chambers, fast timing

Resistive Plate Chambers in barrel

Thin Gap Chambers in endcap Momentum measurement chambers

Monitored Drift Tubes in most of the solid angle

Cathode Strip Chambers in endcap

$\sim 1 \times 10^{6}$ channels

- Related talks

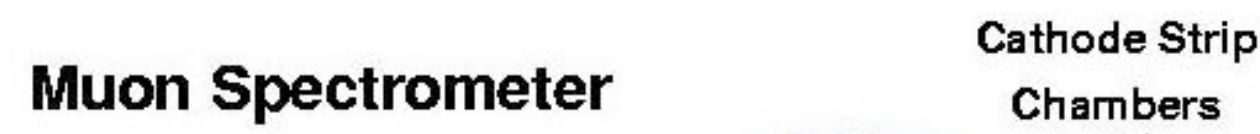

- C. Ferretti (N28), J. Guimarães, J. von Loeben(N30). S.M.Goldfarb, M. Cirilli (N56)

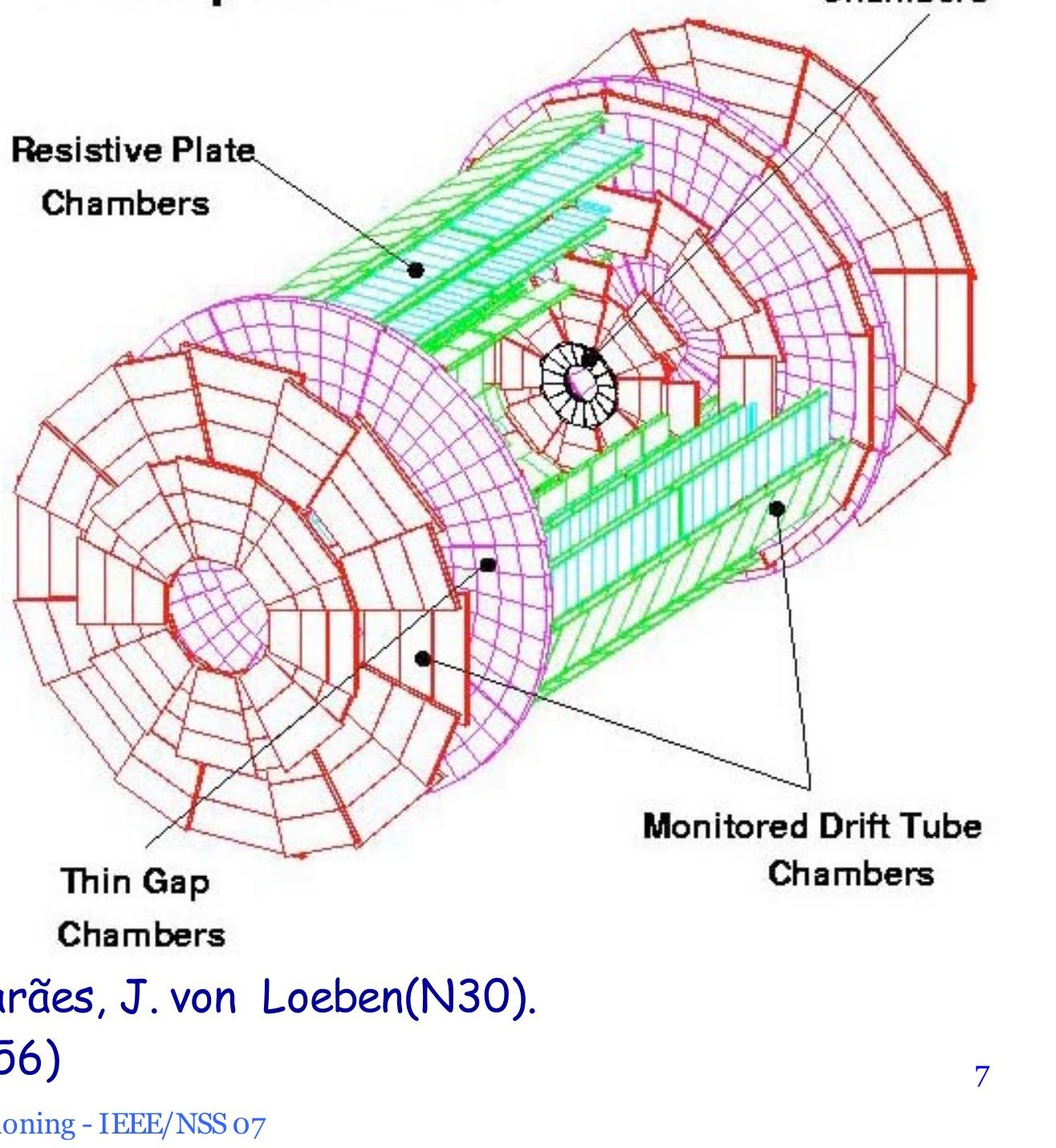




\section{Cosmic ray data}

- Cosmic muon trigger

- TileCal custom board, top-bottom coincidence since 2005

- Muon spectrometer RPC since summer 2007

- Detector integration

- 2005: TileCal standalone

- 2006: combined calo, muon standalone

- 2007: calo+ID+muon

- Organized in "Milestone weeks"M5 ongoing as we speak!

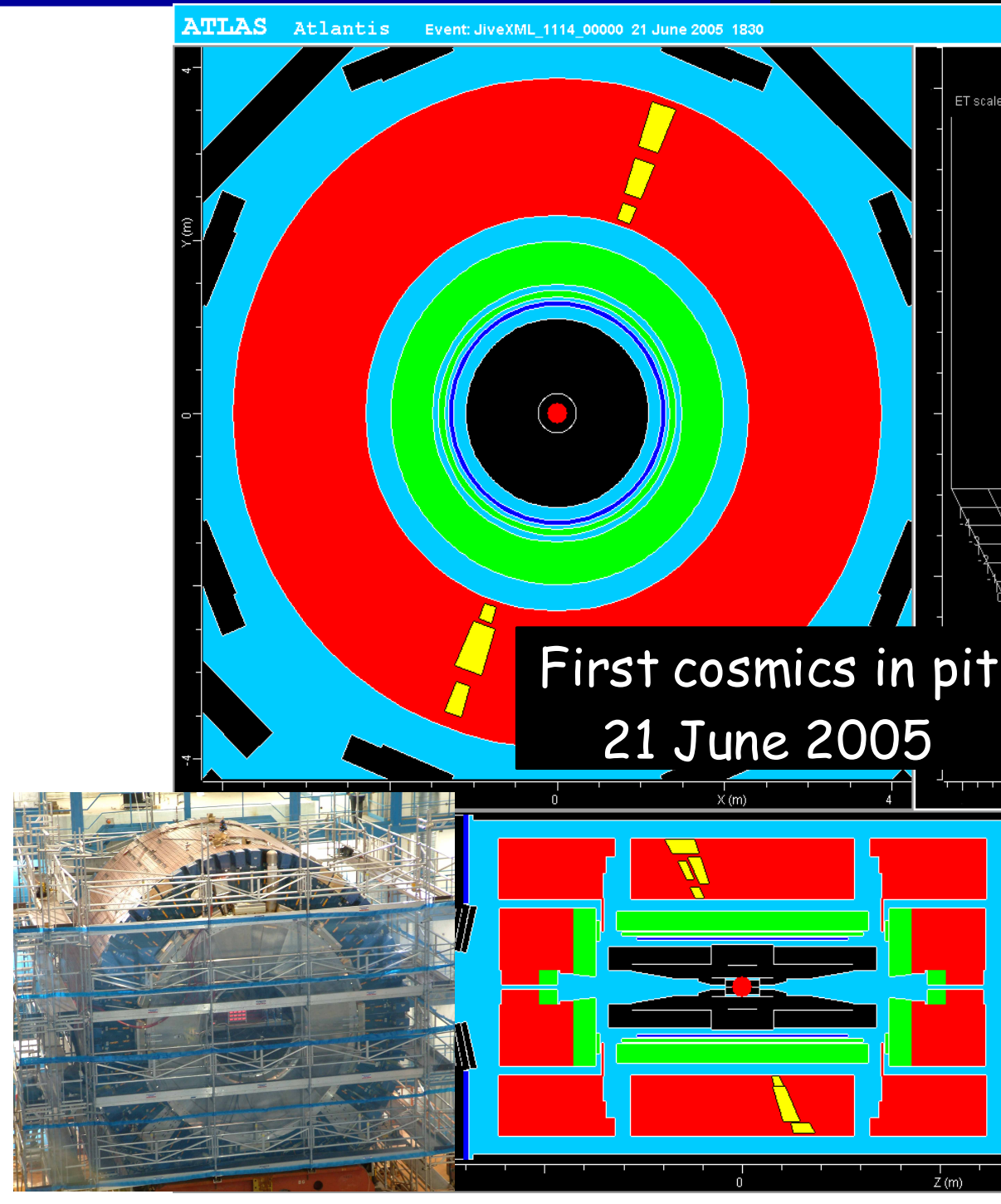

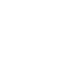


ATLAS software 


\section{ATLAS offline framework}

Athena (adapted from LHCb's Gaudi) is the ATLAS software framework

- Used for

- High-level trigger

- Simulation

- Reconstruction

- Analysis and monitoring

- Flexible structure

- Algorithms and tools

- Event data

- Common services

- DB access, interval of validity

- Transient (Store Gate) and persistent (POOL) data store

- Histogram service,...

Simulation

Cosmic generator GEANT4

Digitization

Detector description Conditions

$D B$
Reconstruction

Integrate different subsystems

Combined algorithms

Data

Monitoring \& Analysis

Event display

Combined monitoring

Subsystem commissioning 


\section{Detector geometry}

- Common detector description for:

- GEANT4 simulation

- Digitization

- Reconstruction

- Stored in OracleDB

- Many different configurations

Commissioning setup

$3 \mathrm{~m}$ gap between barrel and endcap

- Test-beam

- Full ATLAS

- Misaligned ATLAS

- With/without magnetic field Commissioning setups

Gaps due to modules off
Hits in

Tile Barrel

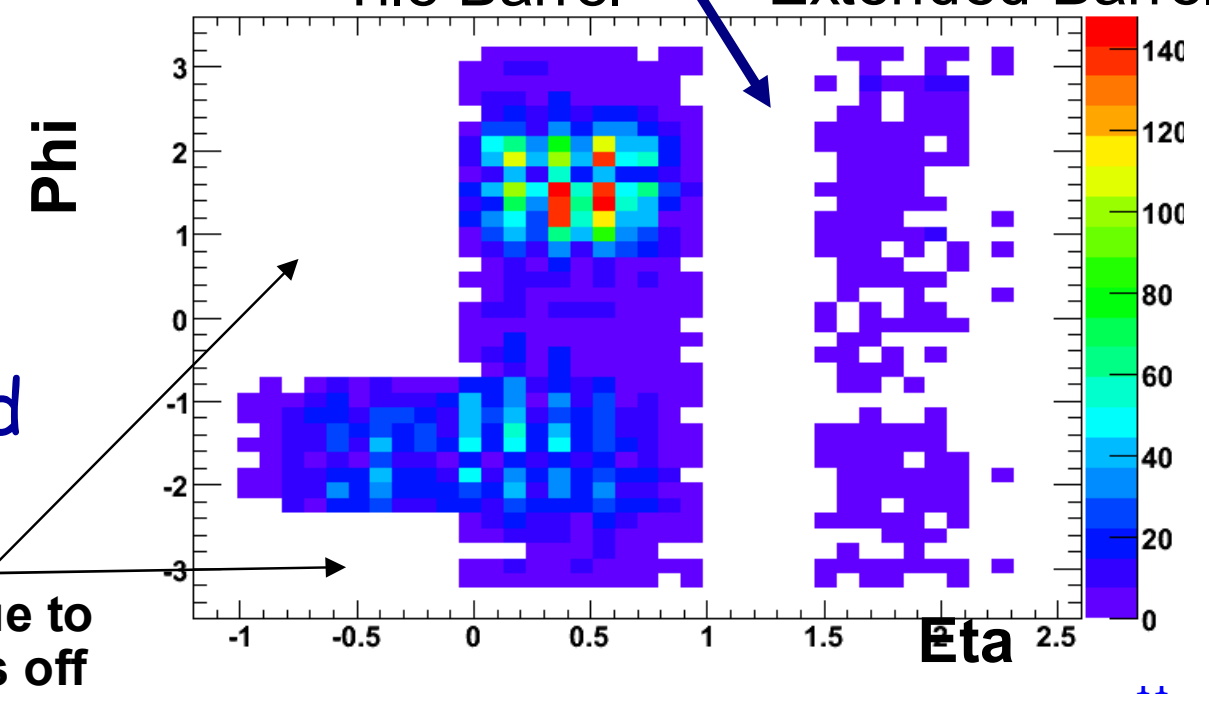




\section{Cosmics generation/simulation}

- Filters applied to generated cosmic rays

- Origin and initial direction

- Simulated tracks must pass in given volume (pixel, TRT, muon volume,...)

- Different handling of time info:

- Hits asynchronous with LHC... Muon position at surface
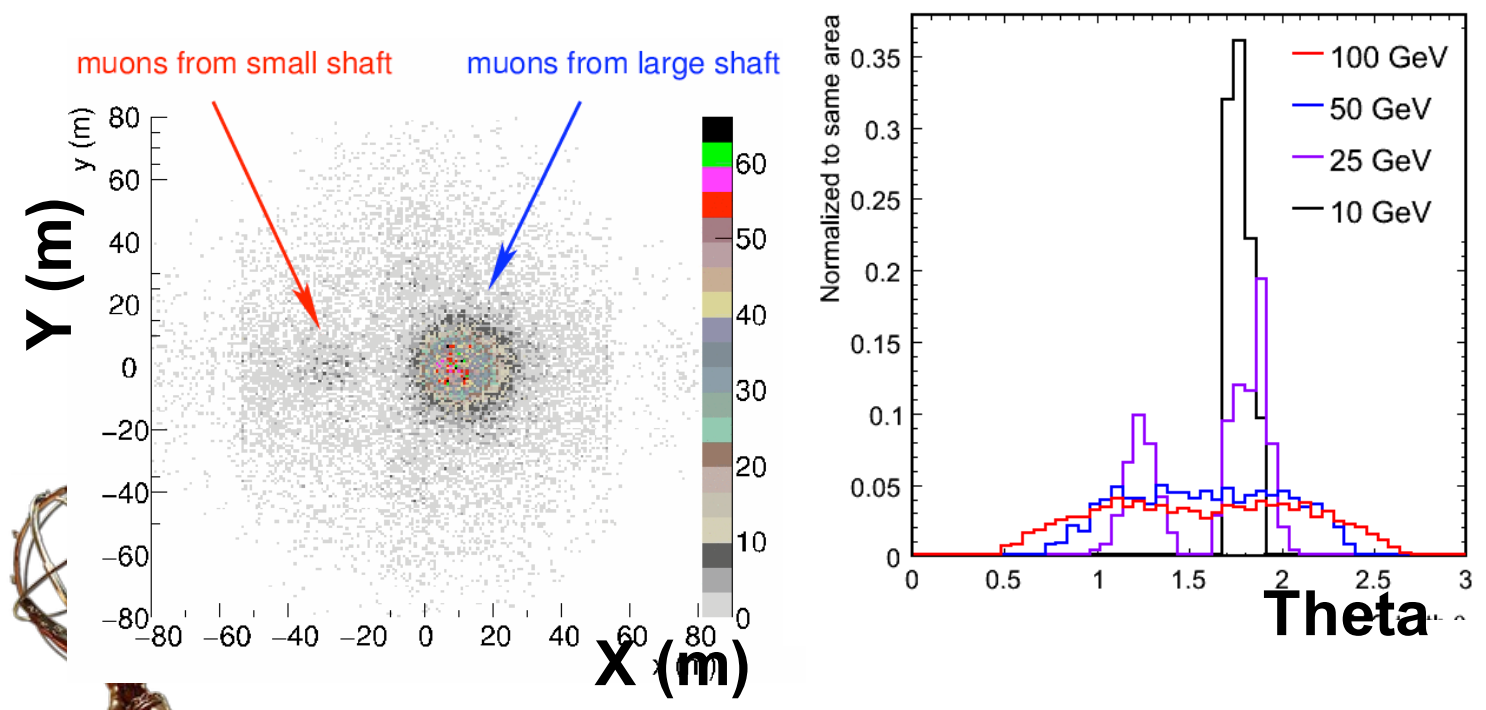

$600 \mathrm{~m} \times 600 \mathrm{~m}$

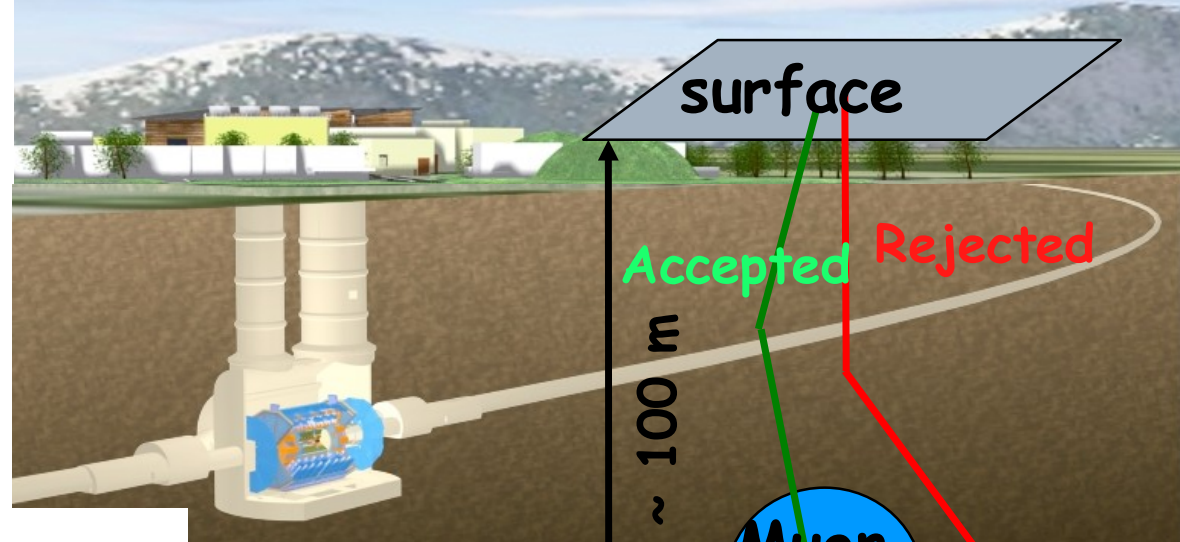

Muon volume

- Low energy muons make it through large shaft

- Rate through TRT: 3 Hz

- Tile: $0.1-0.3 \mathrm{~Hz}$ (higher threshold) 


\section{Cosmics reconstruction}

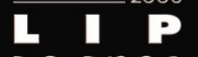

2o anos

Reconstruction by standard and dedicated algorithms. Some adaptations needed:

- Inner Detector and Muon Spectrometer

- Track not pointing to vertex

- More degrees of freedom in fit

- Calorimeters

- Event not synchronized with beam clock

- Phase for optimal filter energy reconstruction not known

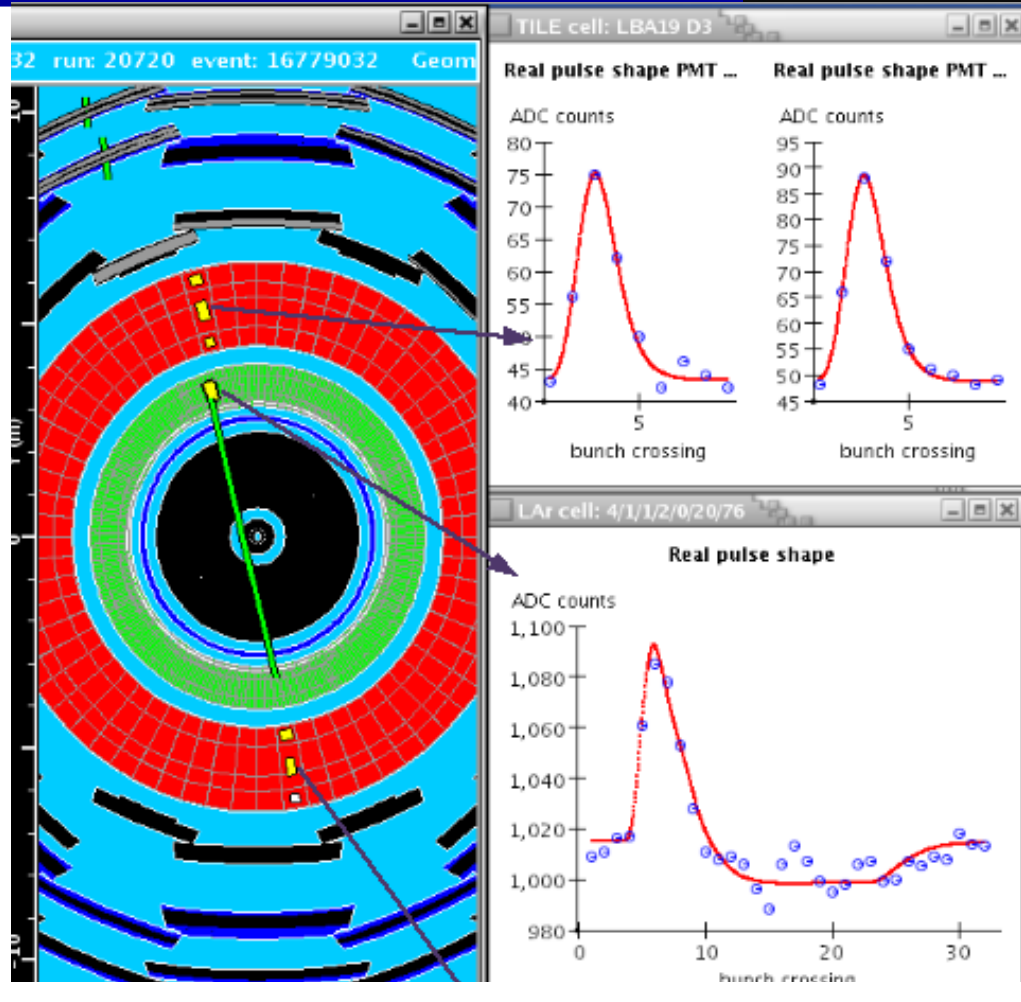

- Solution: iterate LAr reco, or get phase from TileCal 3-parameter signal fit

$E_{\text {cell }} \propto \sum a_{i} \cdot\left(s_{i}-p\right) \quad$ Pulse samples

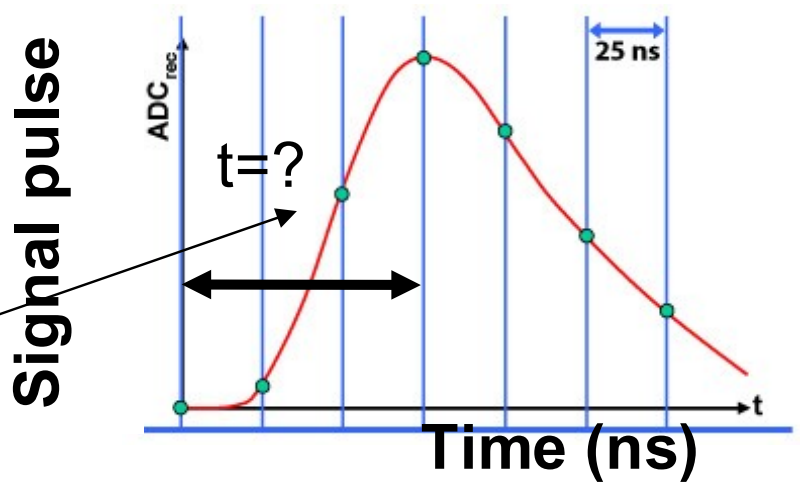




\section{Online Monitoring}

- Partial reconstruction running in online $D A Q$ environment

- Monitoring histograms

- Event display shows the reconstructed objects

- Calorimeter: LAr, Tile cells

- ID: TRT hits and tracks, SCT hits

- Muons: MDT, RPC hits and segments

Updated every $5 \mathrm{~s}$

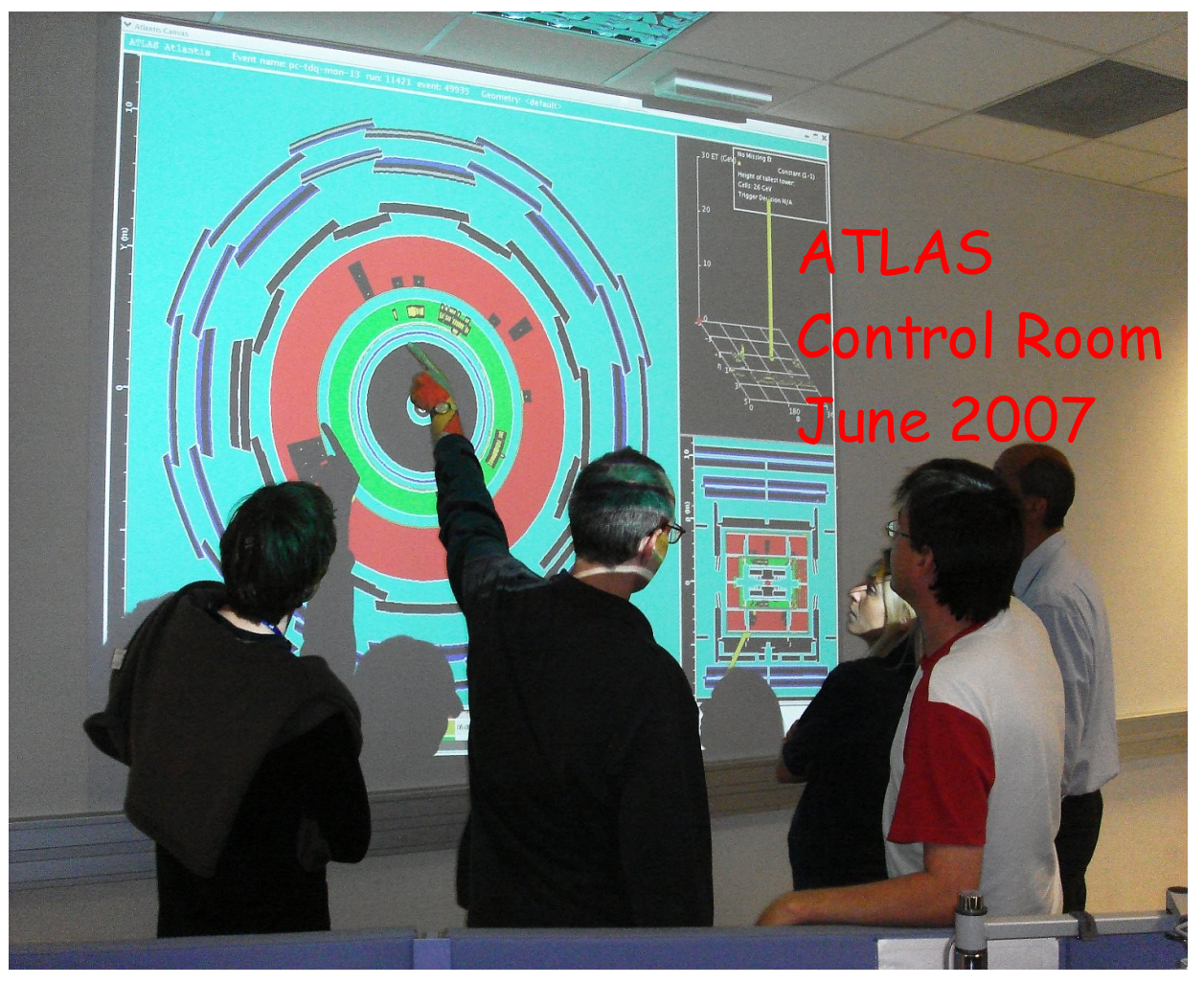




\section{Computing commissioning}

- Processing model

- GRID Tier-O (CERN)

- Prompt first pass processing

- Calibration

- Tier-1's (10 worldwide)

- Process 1-2 months later with better calibrations

- Tier-2's (30+ worldwide)

- Analysis, simulation

- Event data model

- Raw Data Object (1.6 MB/ev)

- Event Summary Data (0.5 MB/ev)

- Reco output, calibration

- Analysis Object Data (0.1 MB/ev)

- Physics-oriented objects
- Commissioning in M4 week (Aug)

- Nightly bug-fix patching

- Tier-0 running on 24 TB of RDO

- Write calo ntuples, ESD ( 0.3 MB/ev)

- Transfer to Tier-1's

- Reprocessed after 1 month

- Less than 1 per mille crashes

- Stressing system by using 25 (instead of 5) LAr samples

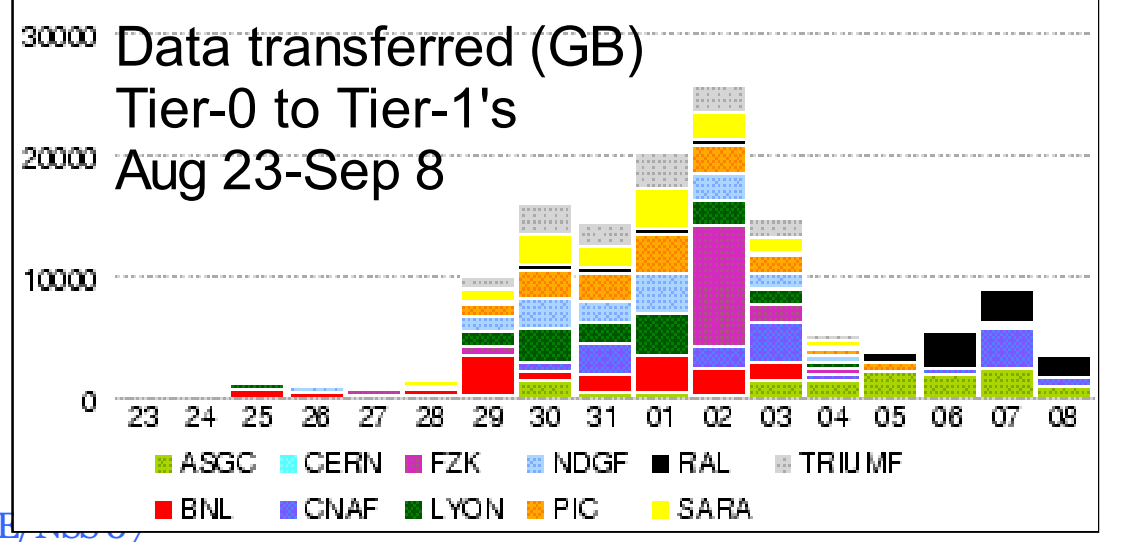




\section{Examples of muon reconstruction}




\section{TileMuonFitter}

- TileCal tool to fit cosmic muon tracks

- Just for commissioning, while muon spect. coverage is partial

- Fits straight line to TileCal cell hits, requires top/bottom, threshold

- Doesn't require projectivity-good for encap as well

- Used to

- Identify "region-of-interest" in LAr and Tile calorimeters

- Provide LAr calorimeter with time phase for optimal filter reconstruction

Provide muon path length

Monitoring

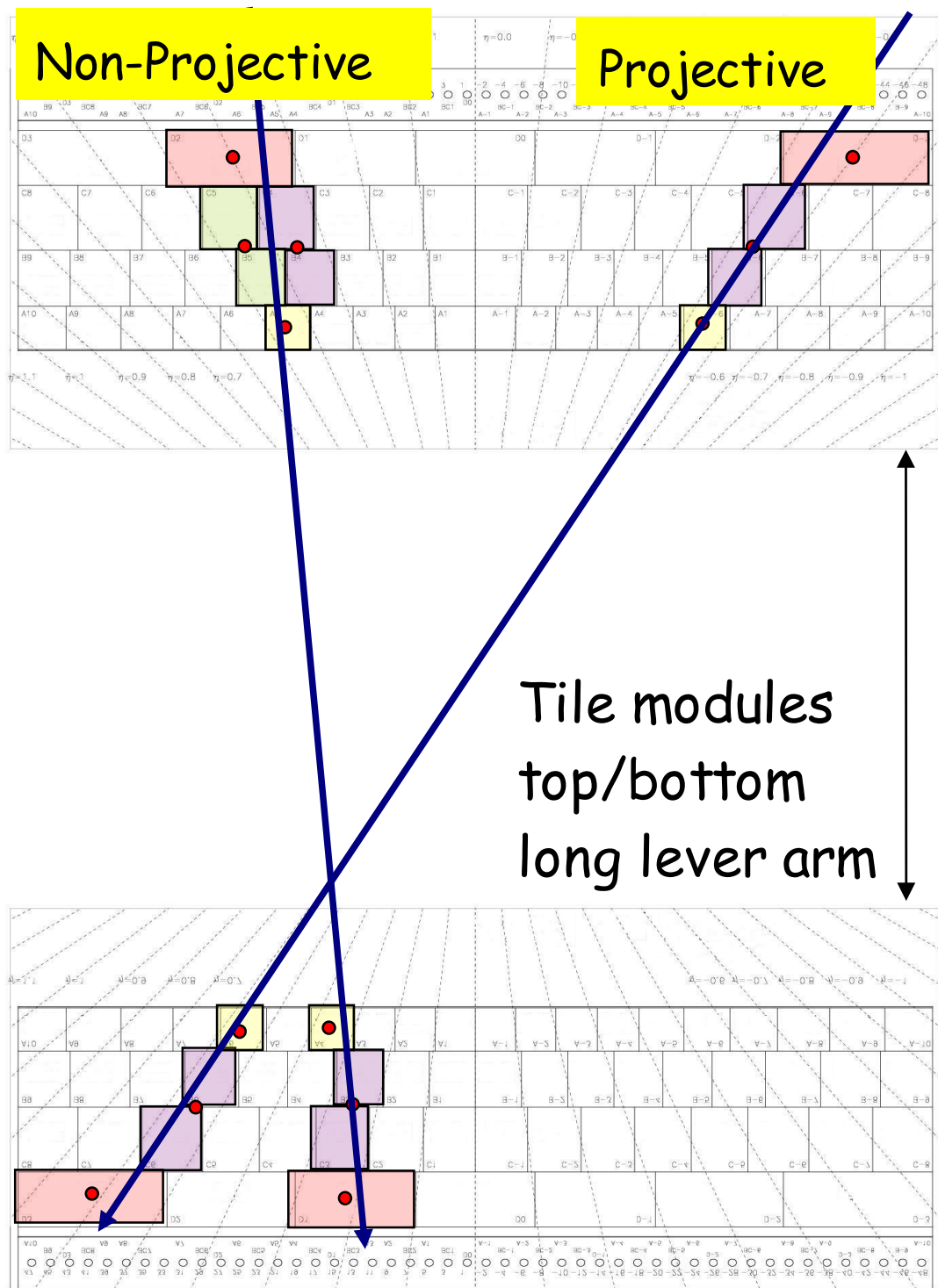




\section{Calorimeter cluster matchina}

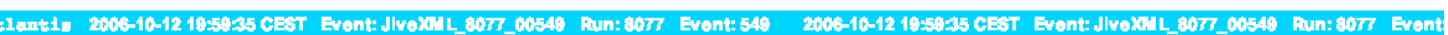
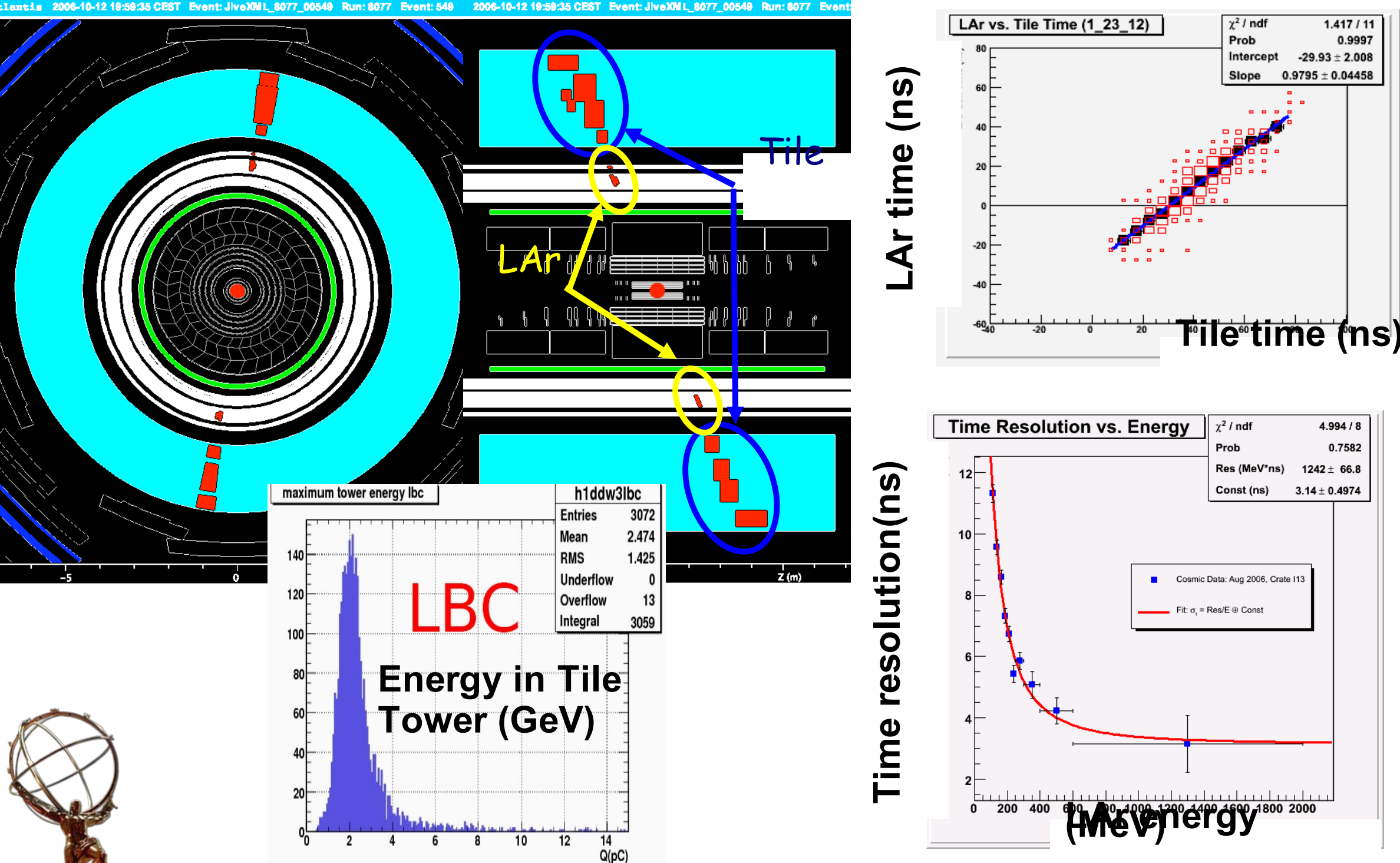


\section{Combined track in real data}

- ID-MUON track

- Combine straight line from MDT segments and TRT tracks

- Another algorithm fits directly from subdetector hits

- Main problem:

- Difficult to include energy loss and multiple scattering effects without magnetic field (no momentum measurement)
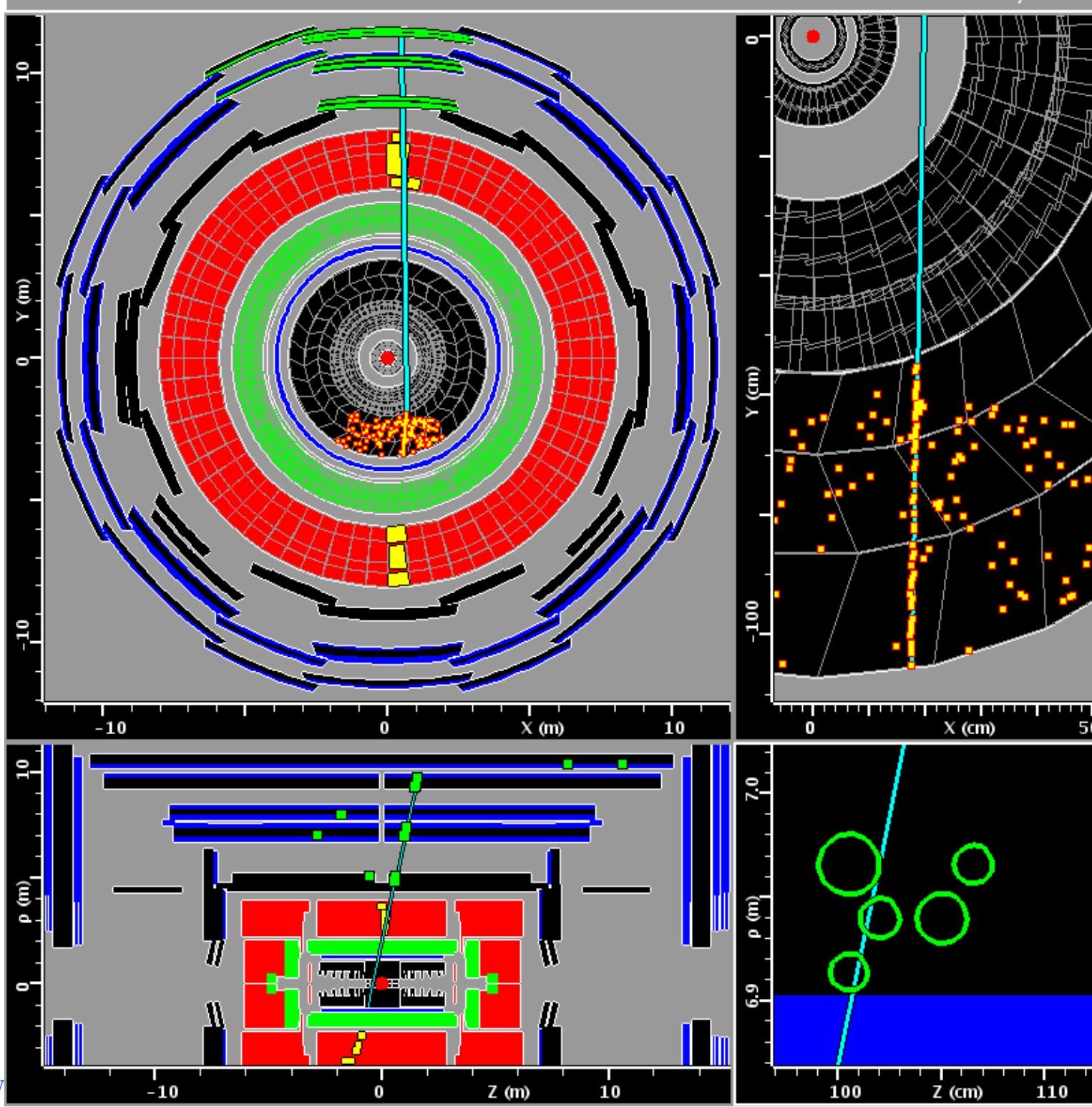


\section{Summary}

- The ATLAS offline software is being commissioned with cosmic rays

- Subdetector commissioning (studies on alignment, uniformity, etc...)

- Ironing out bugs, exercising subsystem integration

- Commissioning will continue

- Integrate more subsystems

- Pixels sub-detector

- L2 trigger

- Magnetic field

- Develop/debug combined algorithms

- Looking forward to LHC collisions! 


\section{Extra alides}

$\mathbf{L} \mathbf{P}$

20 anos 


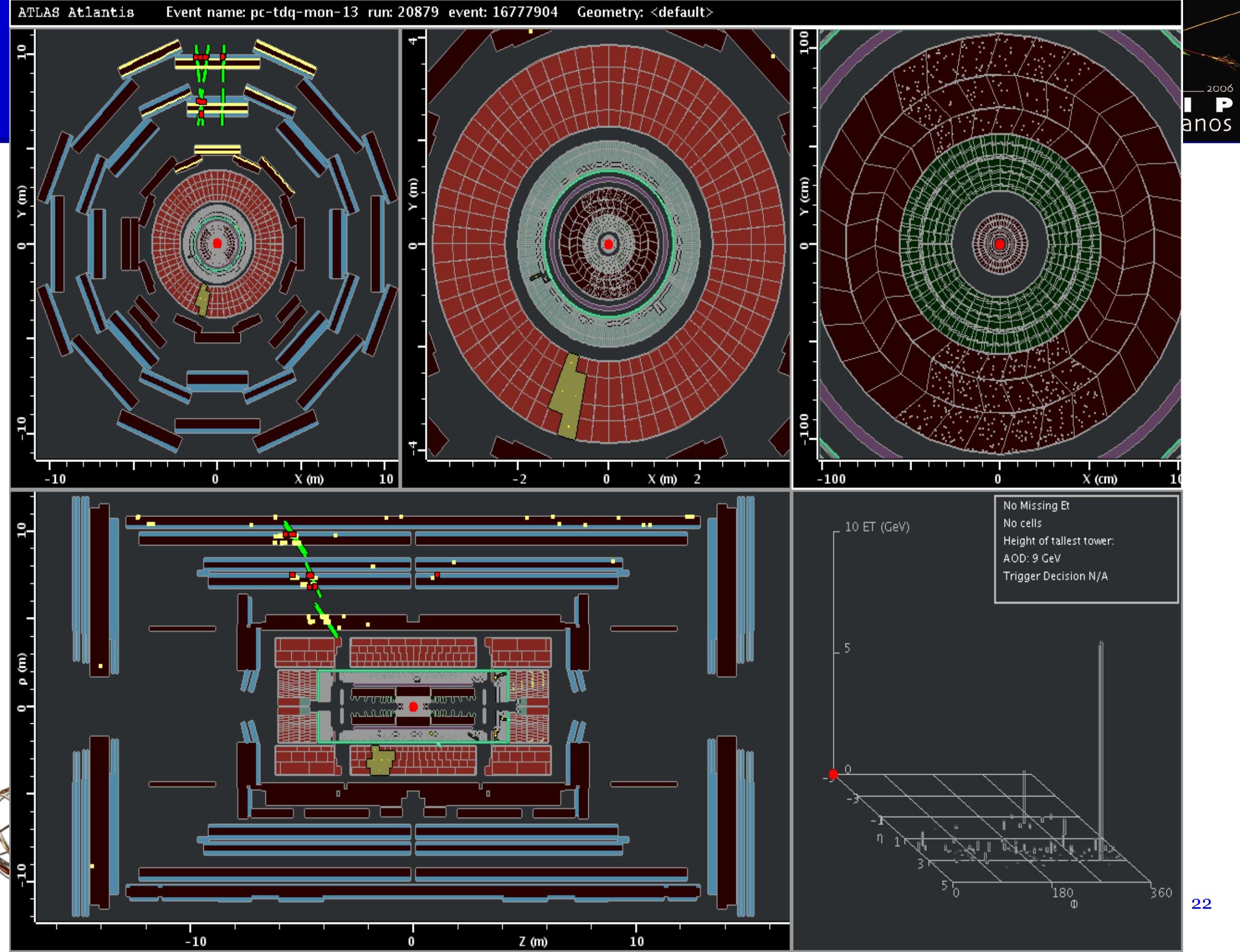




\section{Data analysis}

\section{Example of TRT analys is}

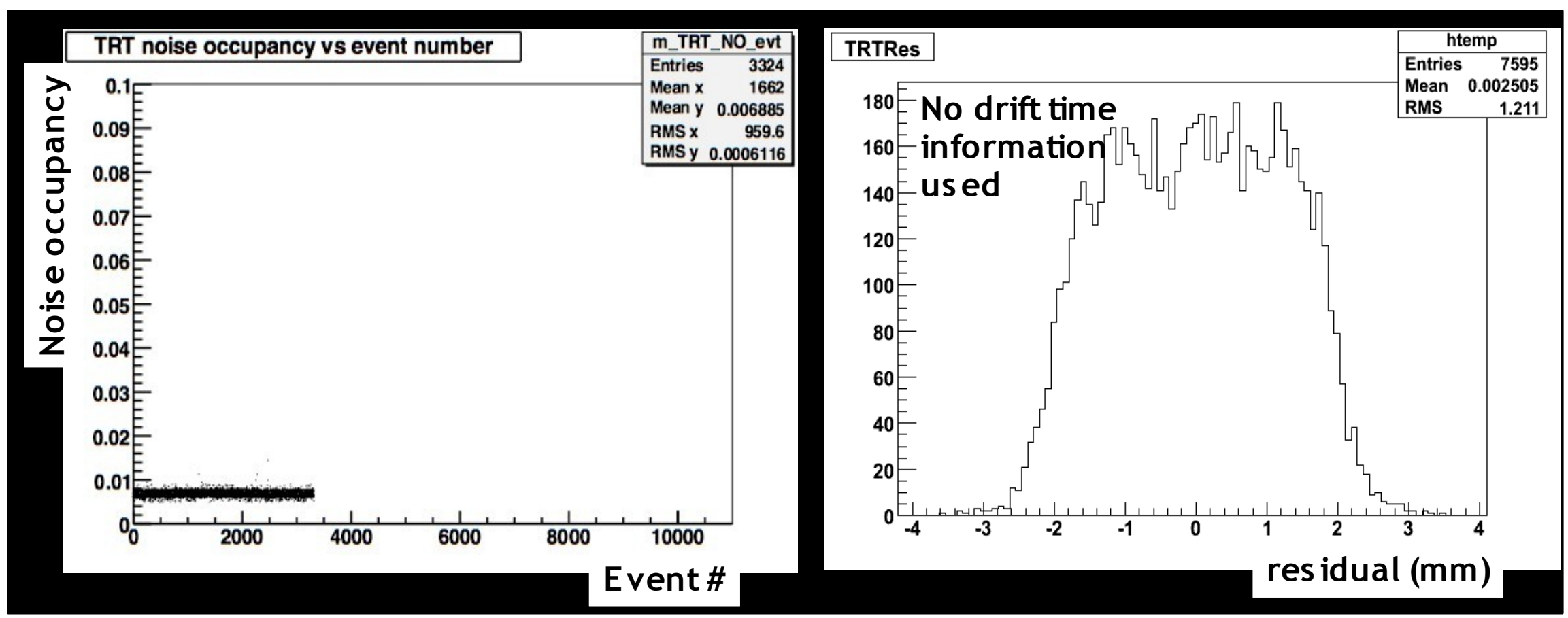

- TRT noise occupancy as expected $(<1 \%)$

- The number of tracks recorded $(\sim 900)$ will not be enough to get a good calibration (track occupancy $<0.03$ tracks/event) 


\section{Data analysis}

\section{Example of Muon analys is}

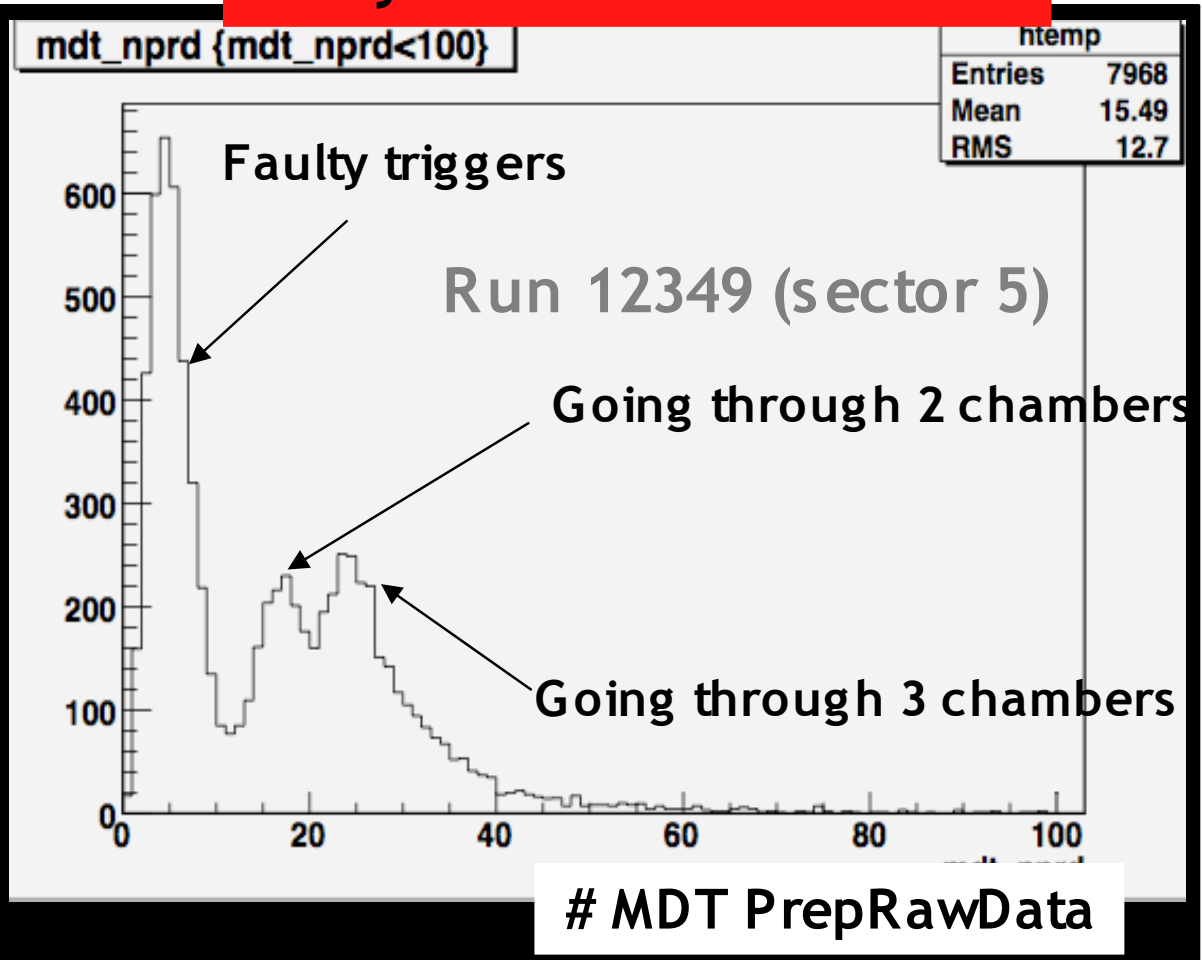

- MDT data (at PRD level) is being studied and compared with previous runs.

- First results with RPC show good data quality.
Sect39 HighPtTrIn

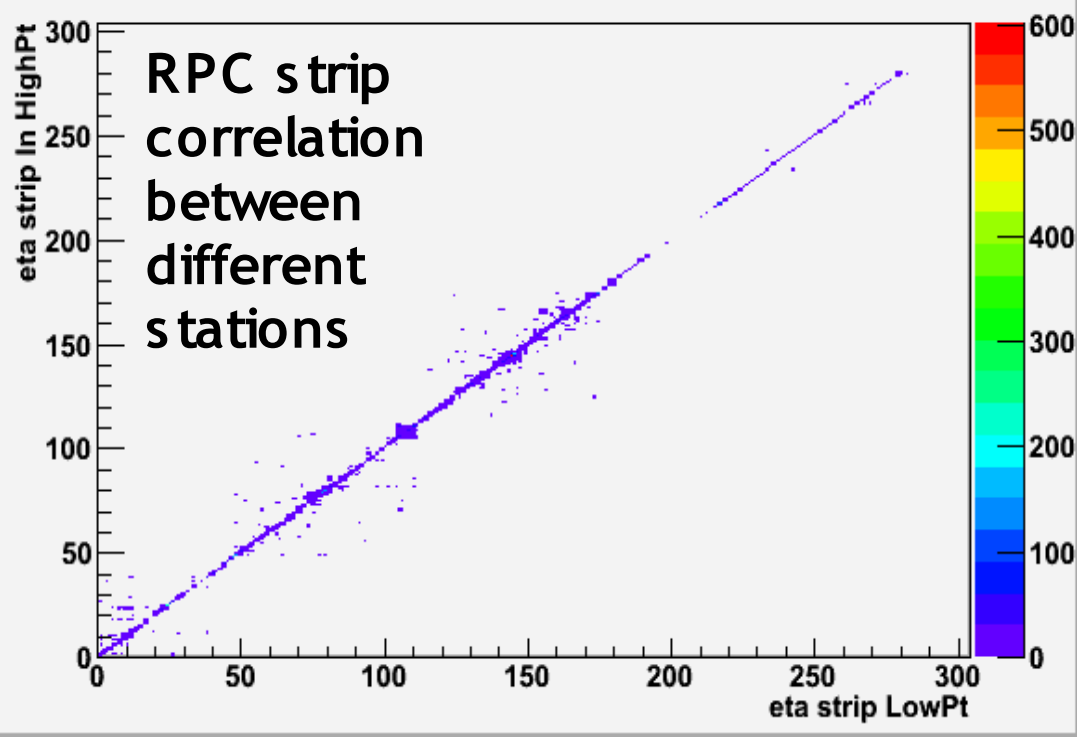

Strip Plane Efficiency

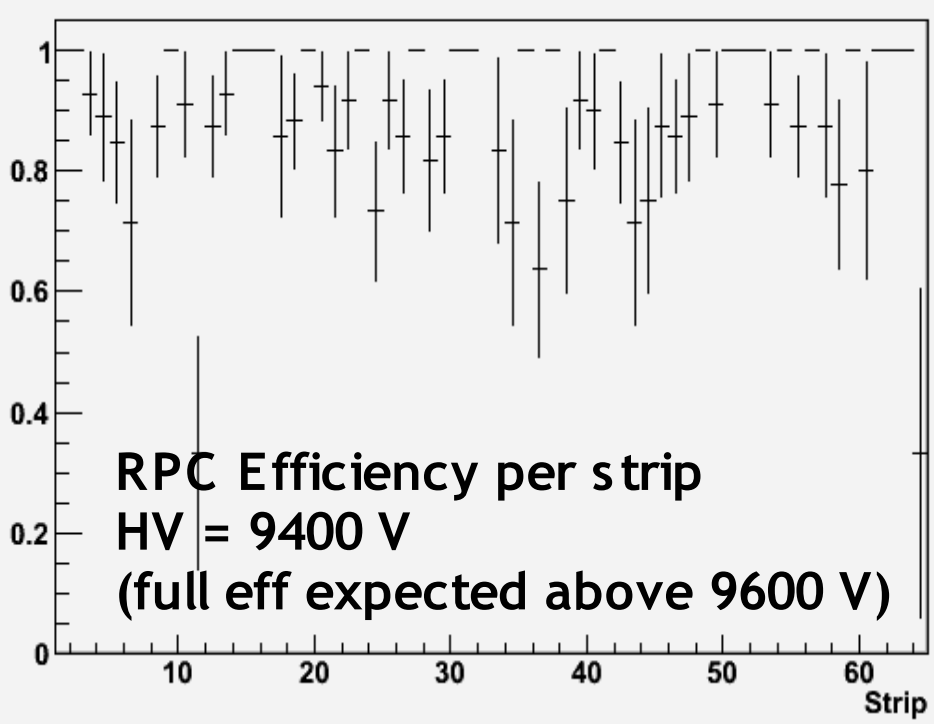




\section{Offline running}

Ex: Extrapolating a track into the calo using CaloTrkMuid

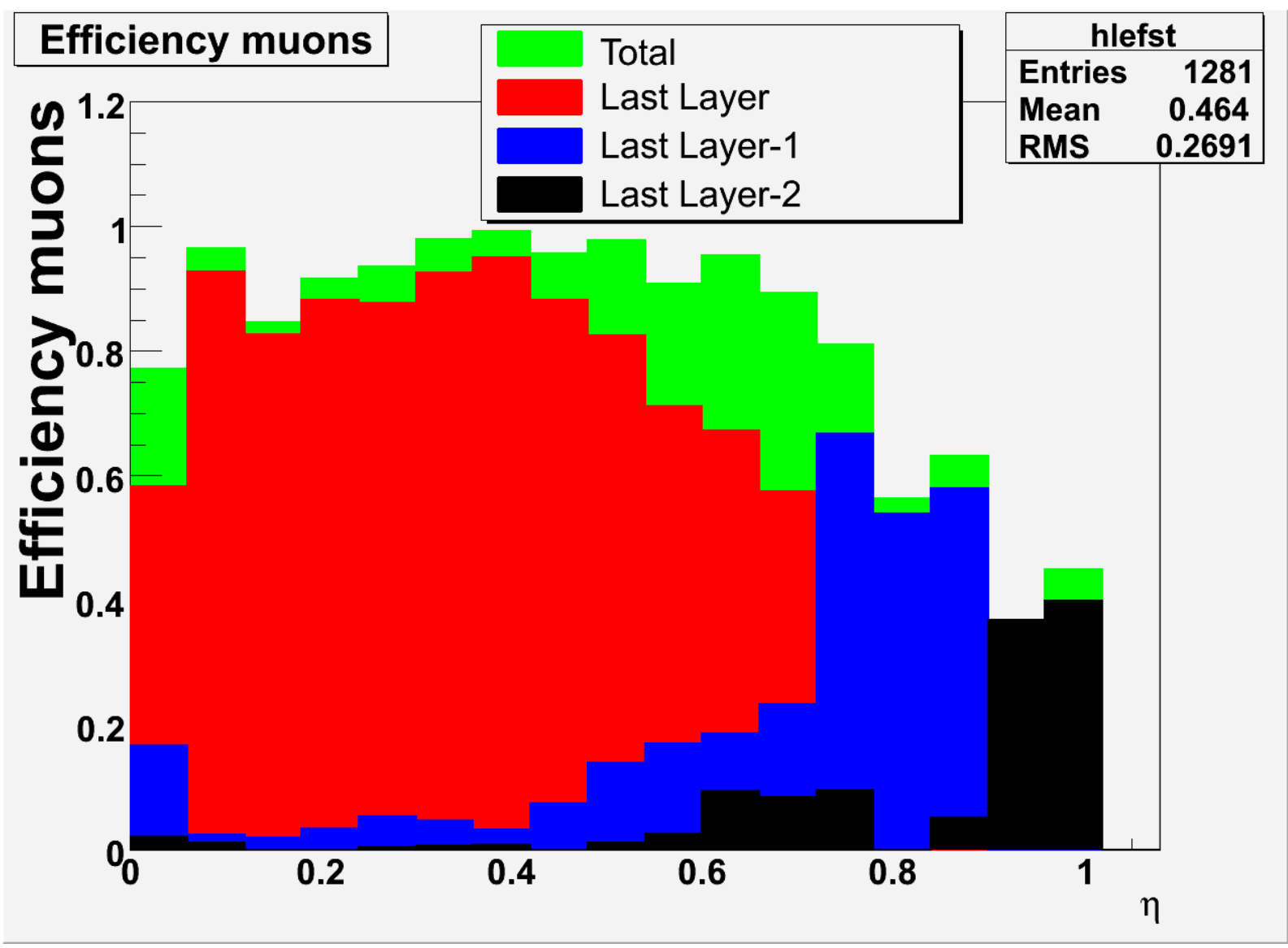

- CaloTrkMuid tags a muon by matching tracks with cells in the $\mathrm{HC}$ al. Plot shows the efficiency us ing simulated data. 


\section{real data}

HEC pulse shape in Atlantis

B efore fix Tile cell energy calibration
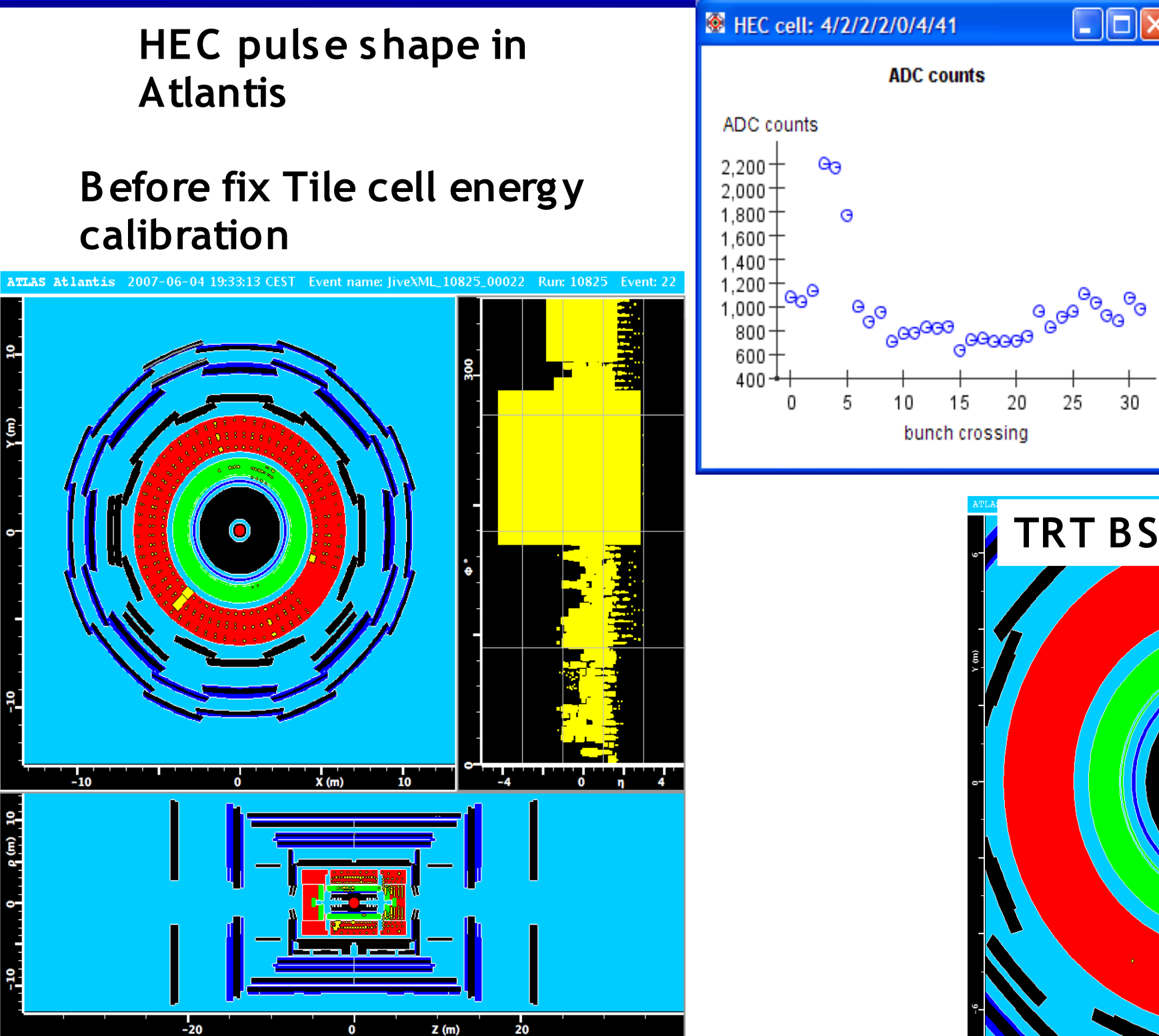

\section{LVL1 Calo decoders}
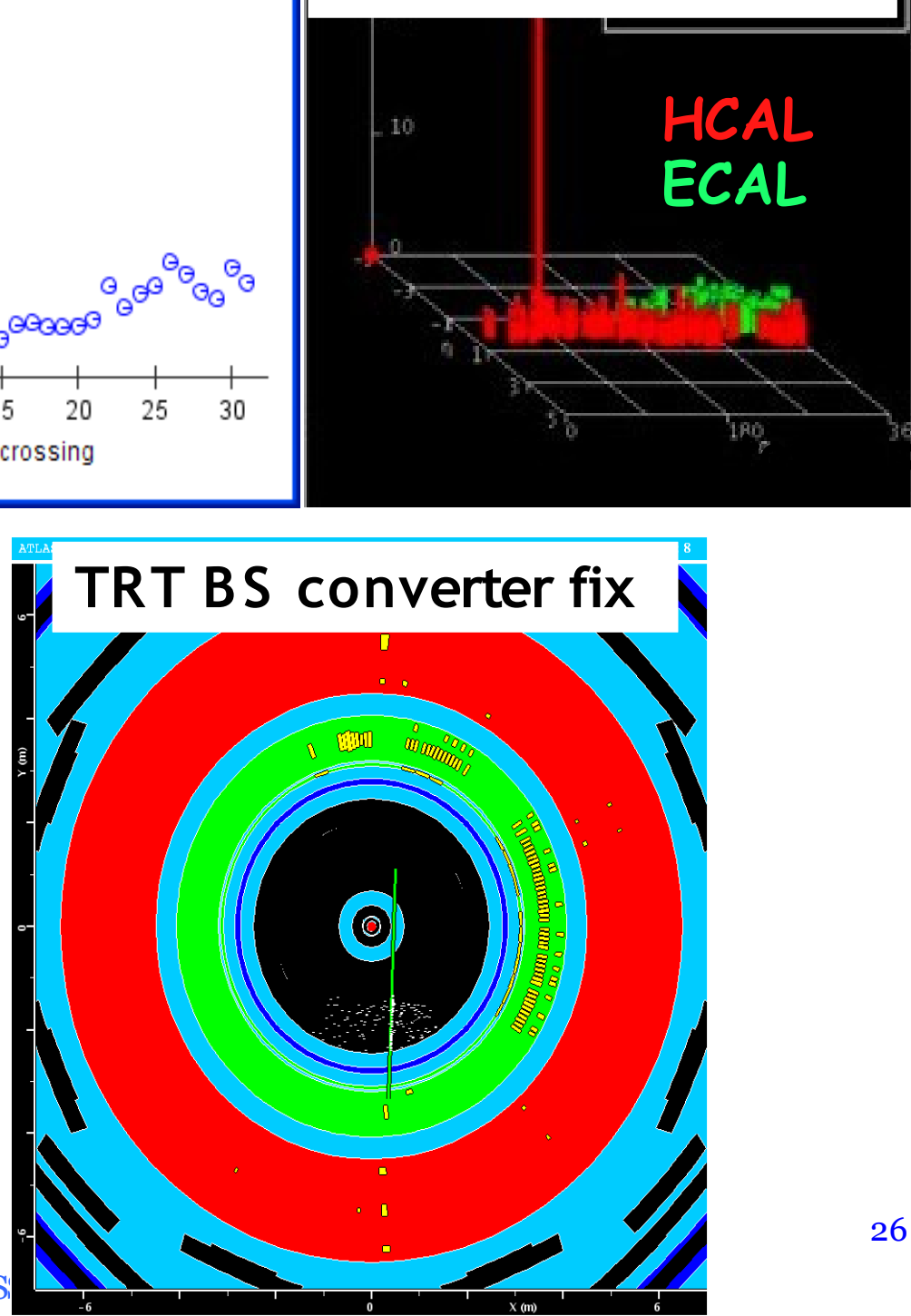